\title{
Modified Bag Models for the Quark Gluon Plasma Equation of State
}

\author{
V. V. Begun, ${ }^{1,2}$ M. I. Gorenstein, ${ }^{1,2}$ and O. A. Mogilevsky ${ }^{1}$ \\ ${ }^{1}$ Bogolyubov Institute for Theoretical Physics, Kiev, Ukraine \\ ${ }^{2}$ Frankfurt Institute for Advanced Studies, Frankfurt, Germany
}

\begin{abstract}
The modified versions of the bag model equation of state (EoS) are considered. They are constructed to satisfy the main qualitative features observed for the quark-gluon plasma EoS in the lattice QCD calculations. A quantitative comparison with the lattice results at high temperatures $T$ are done in the $\mathrm{SU}(3)$ gluodynamics and in the full QCD with dynamical quarks. Our analysis advocates a negative value of the bag constant $B$.
\end{abstract}

PACS numbers: 12.39.Ba 12.40.Ee

Keywords: Bag model, quark gluon plasma, equation of state 


\section{INTRODUCTION}

A transition to the deconfined phase of quarks and gluons, the quark gluon plasma (QGP), is expected at high temperature and/or baryonic density (see, e.g., Refs. [1] and [2] and references therein). In the present study of the QGP equation of state (EoS) we consider the system with zero values of all conserved charges. This is approximately valid for the QGP created in nucleus-nucleus collisions at the BNL RHIC and even better for future experiments at the CERN LHC. Up to now the strongly interacting matter EoS could be only calculated from the first principles within the lattice QCD. These calculations are done for zero or very small values of the baryonic chemical potential. The QGP exists at high temperatures $T>T_{c}$, where the critical temperature $T_{c}$ corresponds to the $1^{\text {st }}$ order phase transition in the pure $\mathrm{SU}(3)$ gluodynamics or to a smooth crossover in the full QCD. The main results for the QCD deconfined matter EoS can be illustrated by the Monte Carlo (MC) lattice results (LR) for the energy density $\varepsilon(T)$ and pressure $p(T)$ in the $\mathrm{SU}(3)$ gluodynamics [3]. The qualitative features of the EoS at $T>T_{c}$ can be summarized as follows. The pressure $p(T)$ is very small at the critical temperature, $p\left(T_{c}\right) / T_{c}^{4}<<1$, and rapidly increases at $T \gtrsim T_{c}$. At high $T$ the system reaches the ideal massless gas behavior $p \cong \varepsilon / 3$, thus, $\varepsilon(T) \cong \sigma T^{4}$. However, the constant $\sigma$ which regulates the high temperature behavior is about $10 \%$ smaller than the Stefan-Boltzmann (SB) constant $\sigma_{S B}$. Both $\varepsilon / T^{4}$ and $3 p / T^{4}$ approach the value $\sigma$ from below. The interaction measure $(\varepsilon-3 p) / T^{4}$, called also the trace anomaly, demonstrates a prominent maximum at $T \cong 1.1 T_{c}$. Note that these properties of the gluon plasma EoS are also valid in the full QCD [4 6 [ $]$.

The bag model (BM) [7] was invented to describe the mass spectrum of the hadron states. Soon after that it was suggested [8] to interpret the bag constant $B$ as the non-perturbative energy density term in the deconfined matter EoS. For several decades, the BM EoS has been used to describe the QGP (see, e.g., Ref. [9]). In its simplest form, i.e. for non-interacting massless constituents and zero values of all conserved charges, the BM EoS reads:

$$
\varepsilon(T)=\sigma_{S B} T^{4}+B, \quad p(T)=\frac{\sigma_{S B}}{3} T^{4}-B
$$

where $\varepsilon$ and $p$ have a simple dependence on $T$ modified by adding the bag constant $B$ ("vacuum pressure"). The SB constant in Eq. (7) is $\sigma_{S B}=\pi^{2} / 30\left(d_{B}+7 d_{F} / 8\right)$, where $d_{B}$ and $d_{F}$ are the degeneracy factors for the massless bosons (gluons) and fermions (quarks and anti-quarks), 
respectively.

The main goal of the present paper is to study the modifications of the bag model EoS. We consider simple analytical parameterizations for the QGP EoS which include a linear and/or quadratic in $T$ terms in the pressure function to satisfy the qualitative properties listed above. The quantitative comparison with the MC LR in the SU(3) gluodynamics [3] and in the full QCD with dynamical quarks [6] will be done in Sections II and III, respectively. The Section IV summarizes the paper.

\section{GLUON PLASMA EQUATION OF STATE}

In this Section a quantitative comparison of the modified versions of the BM EoS is done with the MC LR [3] in the pure $\mathrm{SU}(3)$ gluodynamics. The $\varepsilon / T^{4}$ and $3 p / T^{4}$ were obtained in Ref. [3] by extrapolation to an infinite continuous system. We take the MC values of these extrapolated functions $\varepsilon / T^{4}$ and $3 p / T^{4}$ at the same $T / T_{c}$ points where the interaction measure $(\varepsilon-3 p) / T^{4}$ has been simulated on the finite lattice $32^{3} \times 8$. To determine the parameters of different models discussed below we will minimize the sums of the square deviations at these $T / T_{c}$ points for $\left(\varepsilon-\varepsilon_{M C}\right) / T^{4}$ and/or $3\left(p-p_{M C}\right) / T^{4}$, where $\varepsilon, p$ are the model functions and $\varepsilon_{M C}, p_{M C}$ are the MC LR.

The recent lattice estimate for the pressure at very high temperatures $T / T_{c} \cong 10^{7}$ is still about $3 \%$ below the SB limit [10]. The lowest order perturbative calculations give $\left(\sigma_{S B}-\sigma\right) \propto$ $g^{2}(T) \propto 1 / \ln (T / \Lambda)$. The calculations within the perturbative re-summation scheme [11] are comparable with the $\mathrm{LR}$ at $T=(3 \div 4) T_{c}$ and suggest that the dominant effect of interactions is to turn massless quarks and gluons into weakly interacting quasiparticles. The quasiparticle approach of Ref. [12] (see also recent papers [13] and references therein) treats the system of interacting gluons as a gas of non-interacting quasiparticles with gluon quantum numbers, but with mass $m(T)$ which depends on $T$. The particle energy $\omega$ and momentum $k$ are assumed to be connected as $\omega=\left[k^{2}+m^{2}(T)\right]^{1 / 2}$. The energy density and pressure take then the following form [12]:

$$
\begin{aligned}
& \varepsilon(T)=\frac{d}{2 \pi^{2}} \int_{0}^{\infty} k^{2} d k \frac{\omega}{\exp (\omega / T)-1}+B^{*}(T) \equiv \varepsilon_{0}(T, \omega)+B^{*}(T), \\
& p(T)=\frac{d}{6 \pi^{2}} \int_{0}^{\infty} k^{2} d k \frac{k^{2}}{\omega} \frac{1}{\exp (\omega / T)-1}-B^{*}(T) \equiv p_{0}(T, \omega)-B^{*}(T)
\end{aligned}
$$


where the degeneracy factor $d=2\left(N_{c}^{2}-1\right)$ equals 16 for the $\mathrm{SU}(3)$ gluodynamics. The thermodynamical relation,

$$
T \frac{d p}{d T}-p(T)=\varepsilon(T)
$$

leads to the equation for the function $B^{*}(T)$,

$$
\frac{d B^{*}}{d T}=-\frac{\Delta_{0}(T, \omega)}{m} \frac{d m}{d T}
$$

where $\Delta_{0} \equiv \varepsilon_{0}-3 p_{0}$, and $\varepsilon_{0}, p_{0}$ defined by Eqs. (2,3) are the ideal gas expressions for massive bosons. If the function $m(T)$ is known one can calculate $B^{*}(T)$ from Eq. (5) up to an arbitrary integration constant $B$. For $m=a T$, where $a$ is a temperature independent parameter, the function $B^{*}(T)$ derived from Eq. (5) equals to [14]:

$$
B^{*}(T)=B-\frac{1}{4} \Delta_{0}(T, \omega) .
$$

One obtains the energy density (2) and the pressure (3),

$$
\varepsilon(T)=\sigma T^{4}+B, \quad p(T)=\frac{\sigma}{3} T^{4}-B
$$

which has the form of the BM (11) with constant $\sigma$ equal to

$$
\sigma=\frac{3 d}{2 \pi^{2}} \sum_{n=1}^{\infty}\left[\frac{a^{2}}{n^{2}} K_{2}(n a)+\frac{a^{3}}{4 n} K_{1}(n a)\right] \equiv \kappa(a) \sigma_{S B} .
$$

The $K_{1}$ and $K_{2}$ in Eq. (8) are the modified Bessel functions. The constant $\sigma$ in Eq. (7) includes the suppression factor $\kappa(a)$. For $a \rightarrow 0$, it follows $\kappa \rightarrow 1$, and Eq. (7) coincides with Eq. (11). The function $\kappa(a)$ decreases monotonously and goes to zero at $a \rightarrow \infty$.

The modified SB constant $\sigma<\sigma_{S B}$ allows to fit the high temperature behavior of $\varepsilon(T)$ and $p(T)$. In what follows the BM EoS (7) is considered with $B$ and $\sigma$ being free model parameters. The LR [3] cover the temperature range $(0.89 \div 4.5) T_{c}$. We consider the high temperature phase ("gluon plasma") at $T>T_{c}$, where $T_{c}$ is a point of the $1^{\text {st }}$ order phase transition. To be precise, let us note that we use the MC LR [3] for $T>1.02 T_{c}$ to avoid the uncertainties at $T=T_{c}$ where $\varepsilon(T)$ has a discontinuity in thermodynamical limit. The fit of the MC LR for $3 p / T^{4}$ gives $\sigma=4.62$ and $B=1.56 T_{c}^{4}$, and it is shown by the dashed line in Fig. 17a. One observes a correct behavior, $3 p / T^{4} \cong \sigma$, at high $T$ and an abrupt drop near the critical temperature, $3 p\left(T_{c}\right) / T_{c}^{4} \approx 0$. These features of $p(T)$ are in a qualitative agreement 
with the LR. A quantitative agreement is however unsatisfactory. Moreover, the temperature dependence of $\varepsilon / T^{4}$ calculated by Eq. (7) with $\sigma=4.62$ and $B=1.56 T_{c}^{4}$ appears to be in a qualitative contradiction with the MC LR (see the solid line in Fig. 19).
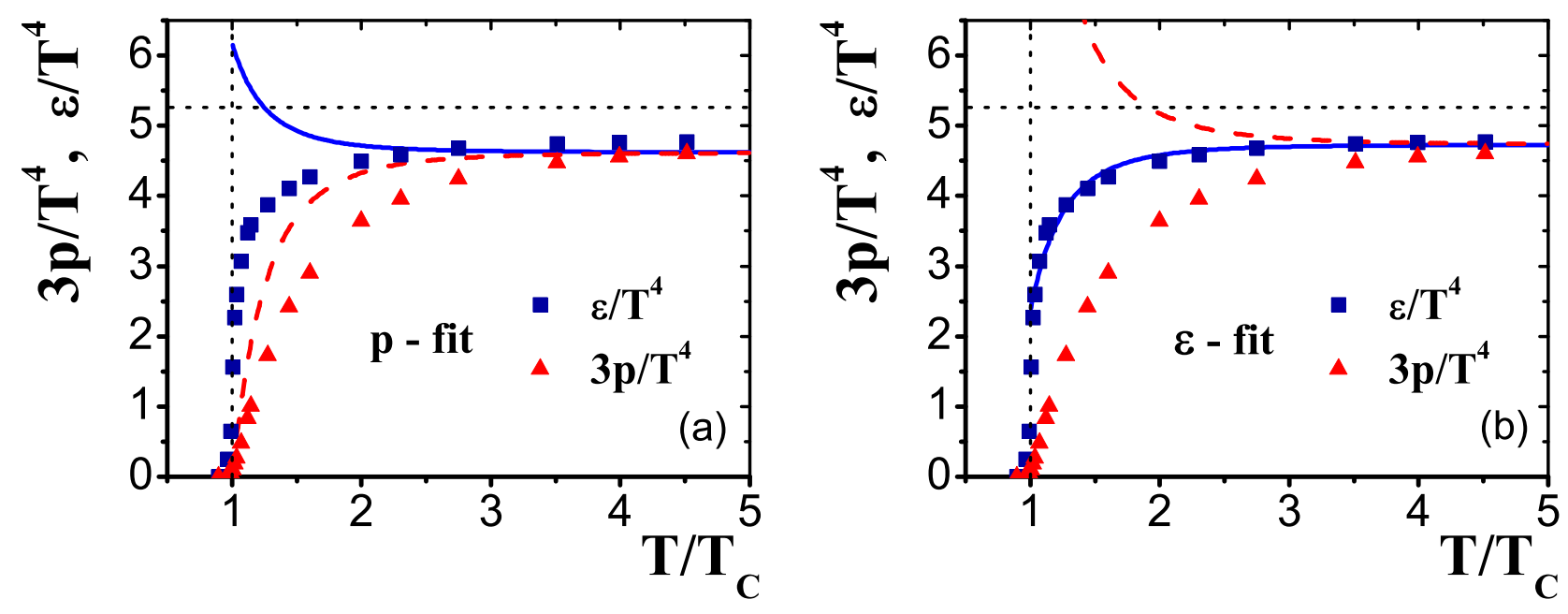

FIG. 1: (Color online) The MC LR for the SU(3) gluodynamics. The $\varepsilon / T^{4}$ (squares) and $3 p / T^{4}$ (triangles) are extrapolated to infinite continuous system [3]. The dotted vertical and horizontal lines correspond to $T / T_{c}=1$ and to the Stefan-Boltzmann constant $\sigma_{S B}=8 \pi^{2} / 15$, respectively. The dashed lines show $3 p / T^{4}$ and solid ones $\varepsilon / T^{4}$ for the BM EoS (7). a: The fit of $3 p / T^{4}$ with the BM EoS (7) gives $\sigma=4.62$ and $B=1.56 T_{c}^{4} . \quad$ b: The fit of $\varepsilon / T^{4}$ with the BM EoS (7) gives $\sigma=4.73$ and $B=-2.37 T_{c}^{4}$.

One can alternatively start from fitting the MC LR for the energy density function $\varepsilon(T)$ with Eq. (77). Unexpectedly, one obtains a rather good agreement with MC LR for $\varepsilon / T^{4}$ admitting negative values of the bag constant $B$. The negative bag constant $B=-2.37 T_{c}^{4}$ and $\sigma=4.73$ needed in Eq. (7) to fit $\varepsilon / T^{4}$ leads, however, to an incorrect behavior of $p / T^{4}$ (see the dashed line in Fig. 10).

A modification of the BM EoS (17) was considered by Pisarski [15]:

$$
\varepsilon(T)=\sigma T^{4}-C T^{2}+B, \quad p(T)=\frac{\sigma}{3} T^{4}-C T^{2}-B
$$

A presence of the $T^{2}$-terms in $p(T)$ and $\varepsilon(T)$ has been further studied in recent papers [16]. For brevity, we will refer to Eq. (9) as the "C-bag model" (C-BM). The fit of the MC LR for $p / T^{4}$ with the C-BM EoS (9) is presented in Fig. 2a. It gives, $\sigma=4.92, B=-0.13 T_{c}^{4}$, 
and $C=1.8 T_{c}^{2}$. One finds an agreement of the C-BM EoS (9) with the LR for $3 p / T^{4}$. In particular, $3 p\left(T_{c}\right) / T_{c}^{4} \approx 0$. However, $\varepsilon\left(T_{c}\right) / T_{c}^{4} \approx 3$, which exceeds the LR.
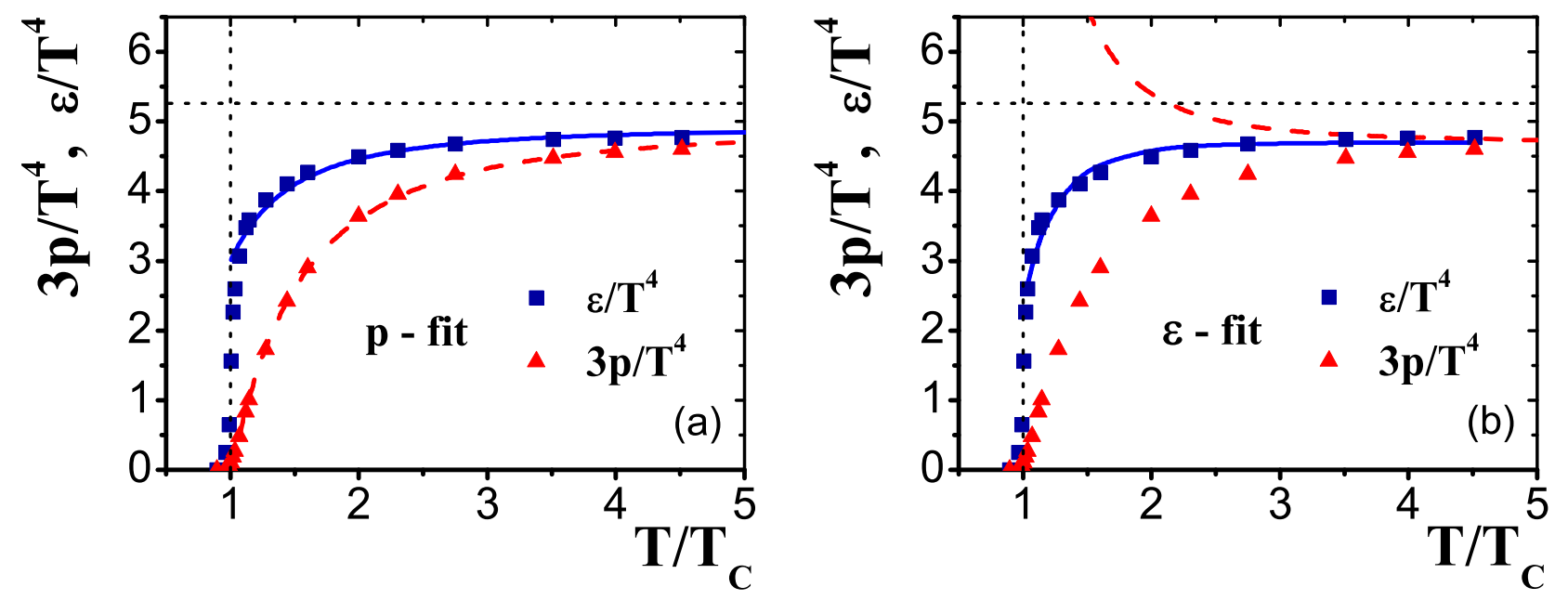

FIG. 2: (Color online) The MC LR [3] are the same as in Fig. 1. The dashed lines show $3 p / T^{4}$ and solid ones $\varepsilon / T^{4}$ for the C-BM EoS (9). a: The fit of $3 p / T^{4}$ with the C-BM EoS (9) gives $\sigma=4.92$, $B=-0.13 T_{c}^{4}$, and $C=1.8 T_{c}^{2} . \quad \mathbf{b}$ : The fit of $\varepsilon / T^{4}$ with the C-BM EoS (9) gives $\sigma=4.69$, $B=-2.64 T_{c}^{4}$, and $C=-0.28 T_{c}^{2}$.

Trying to improve the quantitative agreement with the LR, one may start from fitting the $\varepsilon / T^{4}$ with Eq. (9). One observes indeed a better agreement for $\varepsilon / T^{4}$ with the parameters $B=-2.64 T_{c}^{4}$ and $C=-0.28 T_{c}^{2}$, being very different from those found in the fitting of $3 p / T^{4}$. These new values of $B$ and $C$ lead, however, to a qualitative disagreement of Eq. (99) with $3 p / T^{4}$ LR, as shown by the dashed line in Fig. 2 b.

Comparing the BM EoS (17) and C-BM EoS (9) with the MC LR we have faced the serious challenge. Very different values of model parameters, $B$ for Eq. (7), or $B$ and $C$ for Eq. (9), have been found depending on whether we start from fitting $3 p / T^{4}$ or from $\varepsilon / T^{4}$. By admitting negative values of the bag constant $B$ in Eq. (7) or Eq. (9), one obtains a good fit of $\varepsilon / T^{4}$ in the whole temperature interval $T>T_{c}$, but finds a disagreement with $\mathrm{LR}$ for $3 p / T^{4}$, as seen from Fig. 1b and Fig. 2b. This finding looks contra-intuitive in view that the functions $\varepsilon(T)$ and $p(T)$ are in the one-to-one correspondence to each other due to the thermodynamical consistency equation (4). In Fig. 3 we show the differences between the pressure functions $p(T)$ calculated in the BM EoS (17) or in the C-BM (9), with parameters obtained from the fit 
of $\varepsilon / T^{4}$, and the MC LR for pressure $p_{M C}(T)$. The difference of the pressures is divided by $T_{c}^{4}$. From Fig. 3 one clearly observes a linear temperature dependence of $\left(p-p_{M C}\right) / T_{c}^{4}$. The
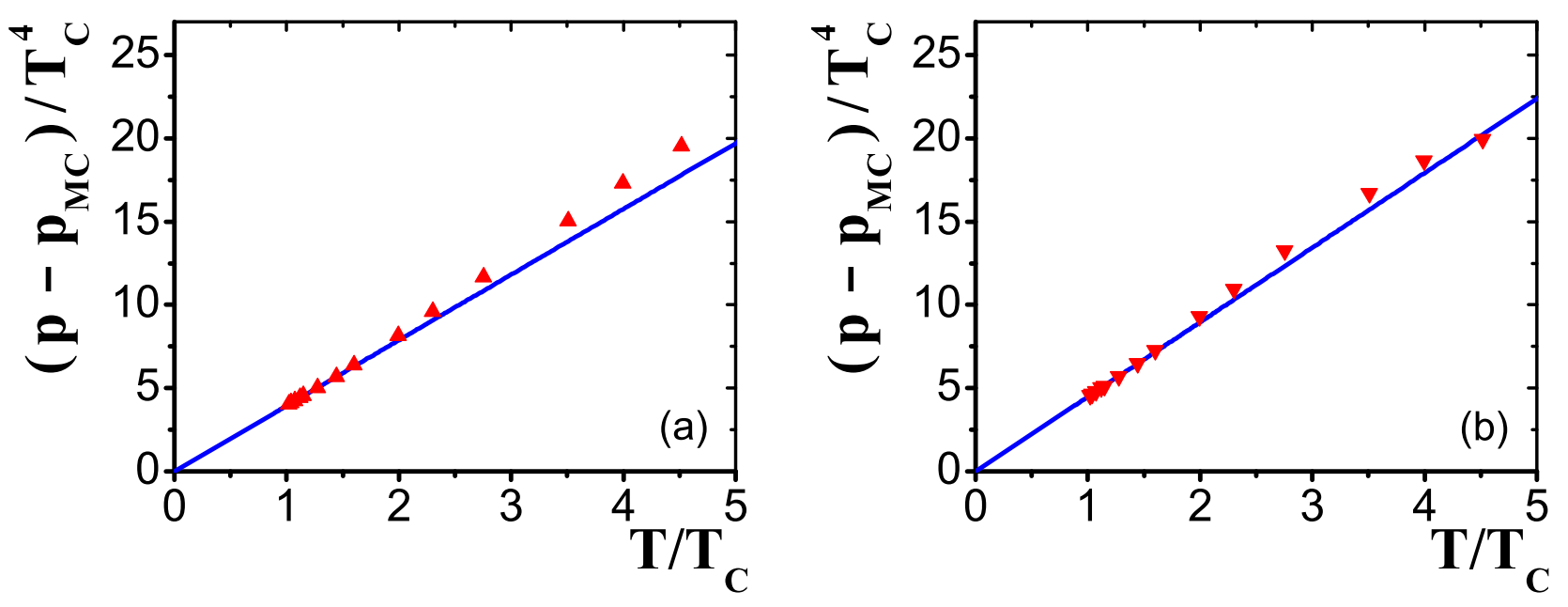

FIG. 3: (Color online) A difference of the model pressure $p$ and the MC LR $p_{M C}$ divided by $T_{c}^{4}$. $\quad$ a: Pressure $p$ is given by the BM EoS (17) with $\sigma=4.73$ and $B=-2.37 T_{c}^{4}$. The Solid line presents the linear function $3.94 T / T_{c}$. b: Pressure $p$ is given by the C-BM EoS (9) with $\sigma=4.69, B=-2.64 T_{c}^{4}$, and $C=-0.28 T_{c}^{2}$. The solid line presents the linear function $4.48 T / T_{c}$.

thermodynamical relation (44) does connect the functions $\varepsilon(T)$ and $p(T)$. This connection is, however, not symmetric in the two directions. If the function $p(T)$ is known, one finds $\varepsilon(T)$ from Eq. (44) in a unique way. However, if the function $\varepsilon(T)$ is known, Eq. (4) is the $1^{\text {st }}$ order differential equation for the function $p(T)$. The general solution of this equation involves an arbitrary integration constant. This results in a linear in temperature term in the function $p(T)$. Thus, for $\varepsilon(T)$ in the form of Eq. (7), a general solution of Eq. (44) for $p(T)$ can be written as follows:

$$
\varepsilon(T)=\sigma T^{4}+B, \quad p(T)=\frac{\sigma}{3} T^{4}-B-A T
$$

The term $-A T$ with an arbitrary constant $A$ corresponds to a general solution of the homogeneous equation $T d p / d T-p=0$ as was noticed in Refs. [17, 18]. For brevity we call the EoS (10) the "A-bag model" (A-BM). The A-BM EoS (10), in contrast to the BM EoS (77) and C-BM EoS (9), gives essentially the same values of the model parameters $\sigma, B$, and $A$ either one starts from fitting $3 p / T^{4}$ or from $\varepsilon / T^{4}$. Figure 4 demonstrates a good agreement of the 

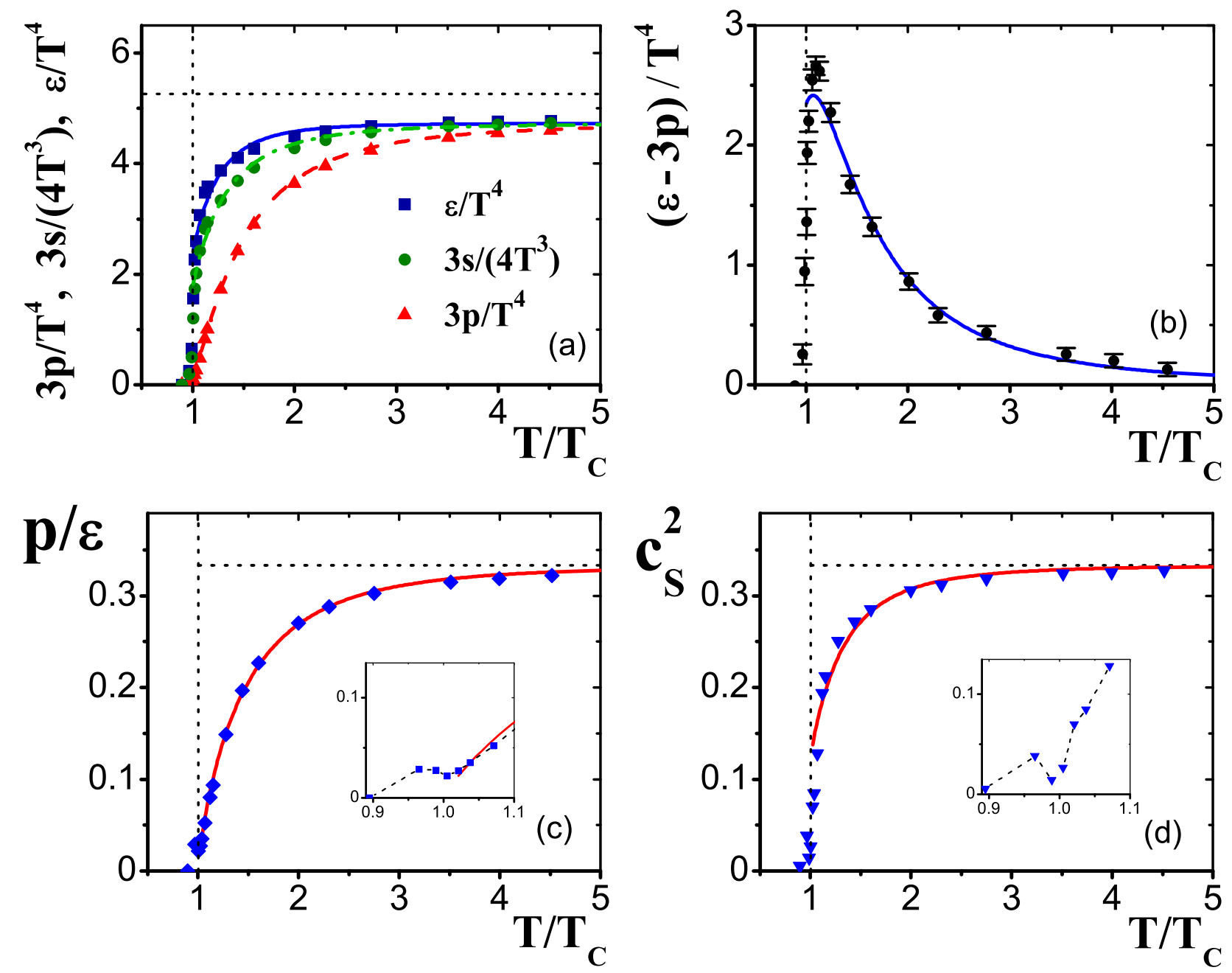

FIG. 4: (Color online) The symbols show the MC LR in the SU(3) gluodynamics [3]. The lines correspond to the A-BM EoS (10) with $\sigma=4.73, A=3.94 T_{c}^{3}, B=-2.37 T_{c}^{4}$. The dotted horizontal lines in figures $\mathbf{c}$ and $\mathbf{d}$ correspond to $p / \varepsilon=1 / 3$ and $c_{s}^{2}=1 / 3$, respectively. $\quad$ a: The squares are $\varepsilon / T^{4}$, triangles $3 p / T^{4}$, and circles $3 s /\left(4 T^{3}\right)$. b: The interaction measure $(\varepsilon-3 p) / T^{4}$. $\quad$ : The ratio $p / \varepsilon . \quad \mathbf{d}$ : The speed of sound squared $c_{s}^{2}$.

A-BM (10) with the MC LR for the thermodynamical functions $\varepsilon / T^{4}, 3 p / T^{4}$, and $3 s /\left(4 T^{3}\right)$ (where $s=(\varepsilon+p) / T$ is the entropy density), interaction measure, $(\varepsilon-3 p) / T^{4}$, the ratio $p / \varepsilon$, and speed of sound squared, $c_{s}^{2}=d p / d \varepsilon$. For the A-BM EoS (10), $(\varepsilon-3 p) / T^{4}$ does not depend on the parameter $\sigma$ whereas the entropy density $s(T)$ does not depend on the bag parameter B. 
Let us consider the EoS which includes both $C T^{2}$ and $A T$ terms,

$$
\varepsilon(T)=\sigma T^{4}-C T^{2}+B, \quad p(T)=\frac{\sigma}{3} T^{4}-C T^{2}-A T-B
$$

referred to as the AC-BM. The standard BM EoS (7) corresponds to $A=C=0$, whereas the C-BM EoS (9) and A-BM EoS (10) correspond to $A=0$ and $C=0$ in Eq. (11), respectively. A comparison of the AC-BM EoS (11) with the MC LR for the $\varepsilon / T^{4}$ and $3 p / T^{4}$ in $\mathrm{SU}(3)$ gluon plasma leads to $C / T_{c}^{2}<<1$. We thus conclude that the AC-BM (111) for the gluon plasma is reduced to the A-BM EoS (10). Note that the lattice study of $\mathrm{SU}\left(\mathrm{N}_{c}\right)$ gluodynamics with $N_{c}=3,4,5,6$, and 8 colors performed in Ref. [22] reveals that $3 p / T^{4}$ and $\varepsilon / T^{4}$ divided by the corresponding SB limits follow essentially the same curves for different $\mathrm{N}_{c}$.

A physical origin of the linear in $T$ term requires further studies. In this connection we remind the famous problem of Gribov copies [19] and his suggestion of the modified gluon dispersion relation, $\omega(k)=\sqrt{k^{2}+M^{4} / k^{2}}$, where $M$ is a QCD mass scale. It was shown in Ref. [20] that at $T>>M$ this dispersion relation gives the SB limit $\varepsilon / T^{4}=3 p / T^{4}=\sigma_{S B}$ and power corrections of relative order $1 / T^{3}$ for $p / T^{4}$ and $1 / T^{4}$ for $\varepsilon / T^{4}$. This also resembles the cut-off $K$ phenomenological model [21] where $\omega(k)=k \theta(k-K)$, i.e. low-momentum gluons are suppressed but high-momentum gluons are effectively free.

\section{QUARK GLUON PLASMA EQUATION OF STATE}

The LR for the realistic equation of state - the QCD with $2+1$ flavors (light $u$-, $d$ - and heavier s-quarks) have been presented by "HotQCD" [4] and "Wuppertal-Budapest" [5, 6] collaborations. We compare the modified BM EoS with the latest LR [6]. The continuum estimates of the LR results for $p / T^{4},(\varepsilon-3 p)$, and $c_{s}^{2}$ in the temperature range $100 \mathrm{MeV}<$ $T<1000 \mathrm{MeV}$ are presented in Table 5 of Ref. [6]. At the highest available temperatures one observes the ideal gas behavior, $p \cong \varepsilon / 3$, but constant $\sigma \cong \varepsilon / T^{4}$ is about $20 \%$ smaller than the $\mathrm{SB}$ constant $\sigma_{S B} \cong 15.63$. This behavior is similar to the case of $\mathrm{SU}(3)$ gluodynamics. At small temperatures, $T=100 \div 140 \mathrm{MeV}$, the LR are expected to be smoothly connected with the thermodynamical functions of the hadron-resonance gas.

In contrast to the pure $\mathrm{SU}(3)$ gluodynamics with a $1^{\text {st }}$ order phase transition between glueballs and gluons, the transition from hadrons to quarks and gluons is a crossover. This smooth transition takes place in the narrow temperature range, $T=150 \div 200 \mathrm{MeV}$ where the energy 

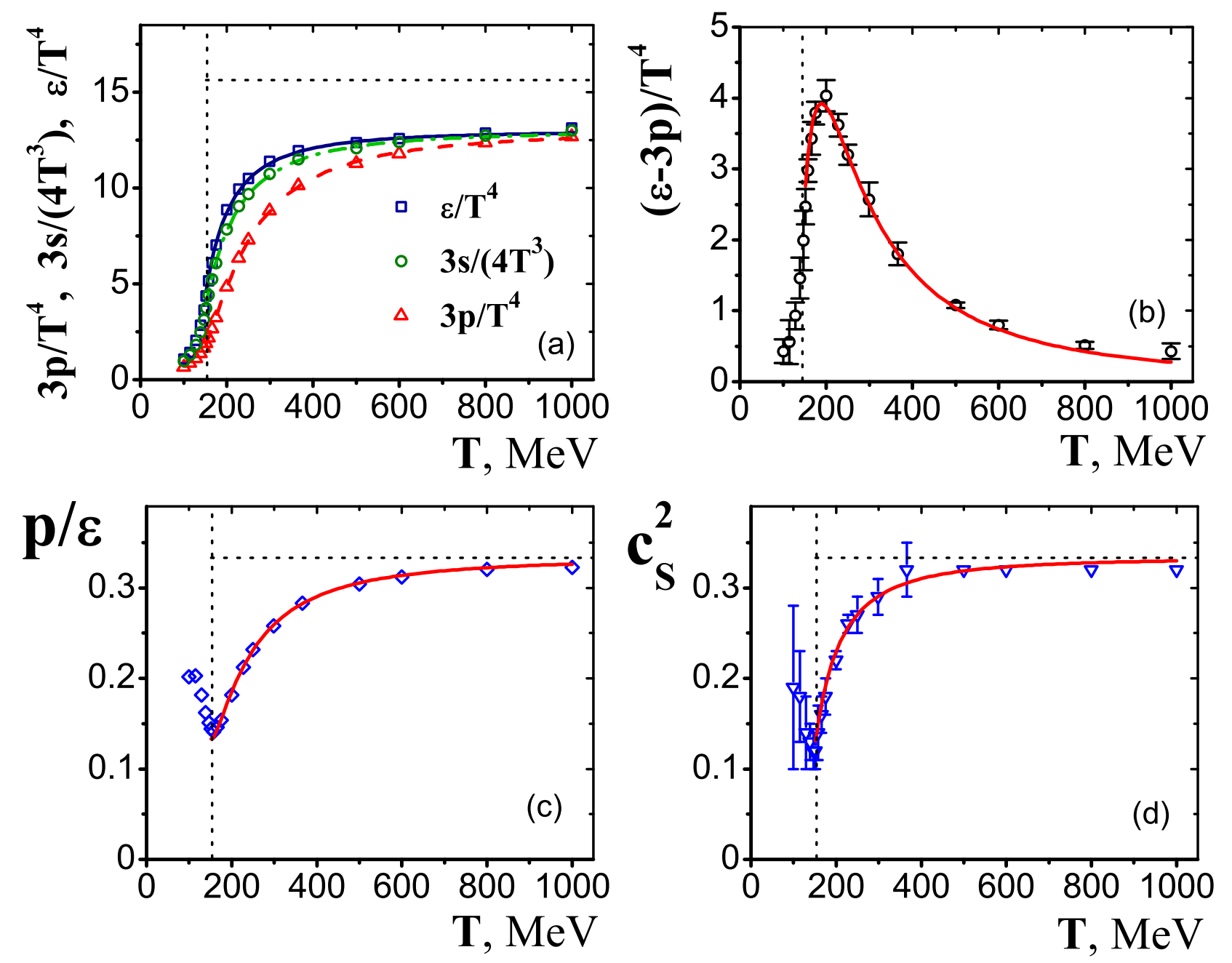

FIG. 5: (Color online) The symbols are the MC LR for the 2+1 QCD EoS [6]. The vertical dotted lines show $T=T_{i}=152 \mathrm{MeV}$ and horizontal lines show the $\mathrm{SB}$ constant $\sigma_{S B} \simeq 15.63$ in figure $\mathbf{a}, p / \varepsilon=1 / 3$ and $c_{s}^{2}=1 / 3$ in figures $\mathbf{c}$ and $\mathbf{d}$, respectively. The lines show the C-BM (9) results at $T>T_{i}=152 \mathrm{MeV}$. The model parameters are $\sigma=13.01, C=6.06 T_{i}^{2}$, and $B=-2.34 T_{i}^{4}$. a: The squares are $\varepsilon / T^{4}$, triangles $3 p / T^{4}$, and circles $3 s /\left(4 T^{3}\right)$. $\quad$ b: The interaction measure $(\varepsilon-3 p) / T^{4}$. $\quad$ : The ratio $p / \varepsilon . \quad \mathbf{d}:$ The speed of sound squared $c_{s}^{2}$.

density increases strongly. Several characteristic temperature points of the crossover transition are presented in Ref. [6]: $T=145(5) \mathrm{MeV}$ at the minimum value of $c_{s}^{2}(T), T \equiv T_{i}=152(4) \mathrm{MeV}$ at the inflection point of $(\varepsilon-3 p) / T^{4}, \quad T=159(5) \mathrm{MeV}$ at the minimum value of $p / \varepsilon, T \equiv$ $T_{\max }=191(5) \mathrm{MeV}$ at the maximum of $(\varepsilon-3 p) / T^{4}$. Non of these temperatures is the critical one, and the model fit of the QGP thermodynamical functions does not depend too much on the 
choice of particular starting point in the range $T=150 \div 200$. However, the hadron-resonance gas expected at low temperatures gives a concave shape of the interaction measure $(\varepsilon-3 p) / T^{4}$, while the LR [6] show a convex shape near the maximum at $T \equiv T_{\max }=191 \mathrm{MeV}$. Thus we use the LR [6] above the inflection temperature $T>T_{i}=152 \mathrm{MeV}$ in our model analysis ${ }^{1}$. This temperature will be also used to present the model parameters.

We start with the AC-BM (11) to fit the $3 p / T^{4}$ and $\varepsilon / T^{4}$ LR for the high temperature QGP phase. The best fit corresponds to negligible values of the linear temperature term, $A / T_{i}^{3}<<1$. Thus, in contrast to our analysis of the LR in the pure SU(3) gluodynamics, the AC-BM (11) is reduced to the C-BM EoS (91) for QGP LR. The found model parameters are equal to: $\sigma=13.01, C=6.06 T_{i}^{2}$, and $B=-2.34 T_{i}^{4}$. A comparison of the C-BM (91) with LR [6] at $T>152 \mathrm{MeV}$ is shown in Fig. 5, It demonstrates a good agreement of the C-BM (9) with the LR for the QGP. In particular, the model leads to the maximum position $T_{\max } \cong 189 \mathrm{MeV}$ and the value of $(\varepsilon-3 p) / T_{\max }^{4} \cong 4$ which are very close to the LR.

\section{SUMMARY}

We have considered the modifications of the bag model EoS. They are constructed to satisfy the qualitative features expected for the QGP EoS. We make also the quantitative comparisons with the MC lattice results for the $\mathrm{SU}(3)$ gluon plasma [3] and for high temperature equation of state with $2+1$ dynamical quarks [6]. Our modification of the bag model equation of state includes the following features: a suppression of the Stephan-Boltzmann constant; linear or quadratic in temperature term in the pressure function; a negative sign of the bag constant. These features are needed to describe the lattice data. The best fit of the LR for thermodynamical functions in SU(3) gluon plasma are found within the A-bag model (10). This model corresponds to: $\varepsilon=\sigma T^{4}+B, p=\sigma T^{4} / 3-A T-B$. A linear in $T$ term in the pressure function is admitted by the thermodynamical relation (4) between $\varepsilon(T)$ and $p(T)$. The expression for the energy density looks formally the same as in the standard bag model (7). A principal difference from the standard bag model is a negative value of the bag constant $B$.

The quantitative comparison with the MC lattice results for high temperature equation of

\footnotetext{
${ }^{1}$ The precise matching of the hadron-resonance gas and LR is beyond the scope of this paper. The discussion of a possible procedure can be found in Ref. [23].
} 
state with $2+1$ dynamical quarks [6] shows the best fit of the thermodynamical functions for the QGP within the C-bag model (9): $\varepsilon=\sigma T^{4}-C T^{2}+B, p=\sigma T^{4} / 3-C T^{2}-B$. This model also requires $B<0$ to fit the lattice data. Note that a negative value of $B$ found for the gluon plasma and QGP does not contradict to the bag model hadron spectroscopy [7] which requires $B>0$ at zero temperature.

Acknowledgments. We thank M. Gaździcki, W. Greiner, V.P. Gusynin, P. Huovinen, L.L. Jenkovszky, O. Linnyk, O. Kaczmarek, E. Megias, L.M. Satarov, H. Satz, and Y. Schröder for fruitful discussions. V.V. Begun thanks the Alexander von Humboldt Foundation for support. This work was in part supported by the Program of Fundamental Research of the Department of Physics and Astronomy of NAS, Ukraine.

[1] E. V. Shuryak, The QCD Vacuum, Hadrons and Superdense Matter, Lect. Notes Phys. 71 (World Scientific, 2004); J. I. Kapusta, Ch. Gale, Finite-Temperature Field Theory. (Cambridge University Press, 2006).

[2] S. Sarkar, H. Satz, B. Sinha (Editors) The Physics of the Quark-Gluon Plasma. Introductory Lectures, Lect. Notes Phys. 785 (Springer, Berlin-Heidelberg, 2010).

[3] G. Boyd et al, Phys. Rev. Lett. 75, 4169 (1995) and Nucl. Phys. B 469, 419 (1996).

[4] M. Cheng et al. [HotQCD collaboration], Phys. Rev. D 81, 054504 (2010) A. Bazavov et al., Phys. Rev. D 80, 014504 (2009). A. Bazavov and P. Petreczky, J. Phys. Conf. Ser. 230, 012014 (2010).

[5] Y. Aoki, et al, Phys. Lett. B 643, 46 (2006); Y. Aoki, et al, JHEP 0906, 088 (2009).

[6] S. Borsanyi et al., [Wuppertal-Budapest Collaboration], JHEP 1009, 073 (2010); S. Borsanyi et al., arXiv:1007.2580 [hep-lat].

[7] A. Chodos, R. L. Jaffe, K. Johnson, C. B. Thorn and V. F. Weisskopf, Phys. Rev. D 9, 3471 (1974).

[8] J. Baacke, Acta Phys. Polon. B 8, 625 (1977).

[9] E.V. Shuryak, Phys. Rep. 61, 71 (1980); J. Cleymans, R.V. Gavai, and E. Suhonen, ibid. 130, 217 (1986).

[10] G. Endrodi, Z. Fodor, S. D. Katz and K. K. Szabo, PoS LAT2007, 228 (2007), arXiv:0710.4197. 
[11] J. P. Blaizot, E. Iancu and A. Rebhan, Phys. Rev. D 63, 065003 (2001); Nucl Phys. A 698, 404 (2002); Quark gluon plasma, World Scientific, Editors Hwa R.C. et al., 60-122 (2004) arXiv:hep-ph/0303185.

[12] M.I. Gorenstein and S.N. Yang. Phys. Rev. D 52, 5206 (1995).

[13] M. Bluhm and B. Kämpfer, Phys. Rev. D 770344004 (2008), ibid. 77, 114016 (2008); F.G. Gardim and F.M. Steffens, Nucl. Phys. A 825, 222 (2009); F. Brau and F. Buisseret, Phys. Rev. D 79, 114007 (2009).

[14] V. V. Begun, M. I. Gorenstein and O. A. Mogilevsky, Ukr. J. Phys. 55, N 9, 1049 (2010), arXiv:1001.3139 [hep-ph].

[15] R. D. Pisarski, Phys. Rev. D 74, 121703 (2006); Prog. Theor. Phys. Suppl. 168, 276 (2007).

[16] E. Megias, E. R. Arriola and L. L. Salcedo, Phys. Rev. D 75, 105019 (2007); O. Andreev, Phys. Rev. D 76, 087702 (2007); E. Megias, E. R. Arriola and L. L. Salcedo, Phys. Rev. D 80, 056005 (2009).

[17] C. G. Kallman, Phys. Lett. B 134, 363 (1984).

[18] M.I. Gorenstein and O.A. Mogilevsky, Z. Phys. C 38, 161 (1988).

[19] V. N. Gribov, Nucl. Phys. B 139, 1 (1978).

[20] D. Zwanziger, Phys. Rev. Lett. 94, 182301 (2005); D. Zwanziger, PoS LAT2005, 191 (2006), arXiv:hep-ph/0509296.

[21] F. Karsch, Z. Phys. C 38, 147 (1988); D. Rischke, M. Gorenstein, A. Schäfer, H. Stöcker, and W. Greiner, Phys. Lett. B 278, 19 (1992).

[22] M. Panero, Phys. Rev. Lett. 103, 232001 (2009).

[23] P. Huovinen and P. Pétreczky, Nucl. Phys. A 837, 26 (2010). 